\title{
Electron-beam-induced uniform elongation of multi-walled carbon nanotube
}

\author{
Imran Khan ${ }^{1,2}$. Jinshen $\mathrm{Lan}^{1} \cdot$ Penggang $\mathrm{Li}^{1} \cdot$ Bing He$^{1} \cdot$ Mengyao Gao ${ }^{1} \cdot$ Shengli Huang ${ }^{1}$
}

(C) Springer Nature Switzerland AG 2019

\begin{abstract}
Multi-walled carbon nanotubes (MWCNTs) respond as brittle materials at room temperature (RT) and fail upon any imposed strain for elongation. By following up our previous study on electron-beam (e-beam)-induced non-uniform elongation of MWCNTs (Khan et al. in J Phys Condens Matter 30:385302, 2018), here, it is illustrated that through moderate irradiation to a high enough intensity e-beam, dramatic uniform elongations can be attained for thin enough MWCNTs at RT, where homogeneous plastic flow of carbon atoms along the tube shells in the tube axis direction takes place with uniform inward diffusion of tube shells. The study also reports well-fitting results for quantitative kinetic relationship of the shrinkage time consequences under nanoscaled curvature, revealing predominant effect of much greater surface energy. These findings are purposeful for processing CNTs and other conventional nanotubes for their utilization in nanodevices and nanotechnology.
\end{abstract}

Keywords Multi-walled carbon nanotube - Uniform elongation · Negative (positive) curvature effect · Electron beam athermal activation effect

\section{Introduction}

Owning to exceptional mechanical strength [2-4] and typical electronic properties [5], carbon nanotubes (CNTs) [6, 7] are extensively used [8] in molecular electronics, optoelectronic and energy storage devices, and other areas of nanotechnology. Regarding to the limitations in their fabrication and assembly, the as-fabricated CNTs may not own the targeted length or diameter. Thus, one may need to plastically (locally) deform them to change their dimensions or configuration. Previously, tensile stress-induced elongations of CNTs (single-walled carbon nanotubes, SWCNTs and multi-walled carbon nanotubes, MWCNTs) have been studied widely [9-14]. In few cases, imposed strains entangled with the bias voltage (heating) have been exploited to elongate them $[9,15]$. Nevertheless,
CNTs are characterized by $\mathrm{sp}^{2}$ hybridized bonded covalent network and flawless rolled graphitic shells, and hence brittle structures under normal conditions [9]. Uniaxial strain at room temperature (RT) may cause plastic elongation, which eventually escorts to the failure of the structure because the structural defects in the typical CNT make it unable to accommodate viscous flow at RT under the imposed deformation rate [10]. This is not a suitable option especially when assembled into devices. Although strain-induced substantial elongation can be achieved with much higher temperature $[9,15]$, heating as high as $2000 \mathrm{~K}$ is not a feasible option, because substantial heating can certainly affect the mechanical properties.

Besides, non-uniform elongation and non-uniform shrinkage of a MWCNT under focused e-beam have been achieved in our previous report [1]. Nevertheless, therein

$\checkmark$ Shengli Huang, huangsl@xmu.edu.cn | ${ }^{1}$ Engineering Research Center of Micro-nano Optoelectronic Materials and Devices, Ministry of Education, Fujian Key Laboratory of Semiconductor Materials and Applications, CI Center for OSED, Department of Physics, Shenzhen and Jiujiang Research Institutes, Xiamen University, Xiamen 361005, China. ${ }^{2}$ Department of Physics, University of Okara, Renala Khurd, Okara 56300, Pakistan. 
[1], probable refilling of the necked region by inward/outward flow of atoms under non-uniform distributions of negative (positive) curvature effect and beam current density over the irradiated segment has made it too intricate to evaluate quantitatively the kinetics of shrinkage and elongation. Although an accurate tracking of the kinetics of uniform elongation and uniform shrinkage of MWCNT and quantitative relationship between the MWCNT shrinkage and nanocurvature effect [16] can be achieved through moderate irradiation or by uniform e-beam current density, the chosen MWCNT should remain straight, smooth, and uniform in the entire irradiation mode to circumvent the effect of non-uniform curvature. Furthermore, such a MWCNT should be thin enough at the very initial stage so that a limited shrinkage could be noted, while irradiating under high enough but non-fluctuating beam current density for an adequate irradiation period. To date, it seems hard to optimize such conditions and to find such a report.

To follow up this [1], here, we demonstrate that the MWCNTs can be allowed to elongate and shrink uniformly to a certain minimum under electron beam irradiation, when the CNTs (MWCNTs) in question are straight, smooth, and uniform in the entire mode, and the current densities of energetic electron beams (e-beams) in transmission electron microscope (TEM) can be kept high enough (optimized) and uniform (minimum fluctuation) for a feasible irradiation period. Mechanisms underlying the structural evolution with quantitative tracking of the MWCNT shrinkage process are also explored in detail.

\section{Experimental}

MWCNTs were fabricated in a floating catalyst apparatus and treated ultrasonically in ethanol. They were dropped onto holey carbon films for TEM studies. In situ irradiation experiments were performed at ambient RT in FEI Tecnai F-30 field emission gun TEM operating at $300 \mathrm{kV}$, equipped with a condenser with correction of the spherical aberration. Small segments of MWCNTs were exposed to focus electron beam of $200 \mathrm{~nm}$ diameters and irradiated under uniform beam current densities of $160 \mathrm{~A} \mathrm{~cm}^{-2}$. The typical, $300 \mathrm{kV}$, e-beam can easily pass through [17] MWCNT diameter. To prevent the influence of support, individual, straight and clean MWCNT segments, protruding into the free space (micro-holes) of the supporting carbon film were irradiated under focused e-beam. To collect micrographs, beam current density was periodically reduced to 200 times weaker than that of irradiation. This increased the imaging contrast of MWCNTs and reduced the radiation damage to a negligible value as well. To examine structural evolutions, micrographs were collected after each succeeding irradiated dose that normally took $15-30$ s to return to the irradiation conditions. The changes in diameter and length after each succeeding irradiated dose of the e-beam were shown sequentially in Fig. 1. All the structural changes were measured between the two yellow dots marked across the MWCNT axis, which were traced by following the relevant feature points along the MWCNT length, shown via black arrows in Fig. 1 and assisted by Fig. 2. The MWCNT diameter was averaged after each dose by calculating from different representative sites. The axis line of the MWCNT between the two yellow marks was considered to measure the length. The error bars in Fig. 3 represented the integrative deviations from the mean values. Furthermore, to prevent projection/shortening in the diameter/length, MWCNT was kept under focus by continuous tilting thus always keeping the position of MWCNT axis normal to the e-beam as well. It is noteworthy that $300 \mathrm{kV}$ e-beam produces negligible heat in MWCNTs due to their extremely high ratio of surface/ volume as the recent research shows [18], and e-beam effect is nonthermal. Thus, it is expected that MWCNT essentially keeps itself at RT under irradiation.

\section{Results and discussion}

Figure 1 illustrates TEM series map of the representative evolution process in a MWCNT induced by subsequent irradiation up to $550 \mathrm{~s}$. Figure $1 \mathrm{a}$ shows the length and diameter of a single, straight, and local segment of MWCNT with shells $\sim 10$ prior to irradiation that passes over the bundle of tubes and hangs into the free space. The initial length and diameter of the MWCNT are, respectively, measured to be $37 \mathrm{~nm}$ and $14 \mathrm{~nm}$ marked with the two featured yellow marks and traced via the two black arrows. Figures $1 \mathrm{~b}-1$ demonstrates ongoing evolution in the MWCNT structure monitored systematically in situ via TEM. Without any breakage under no imposed strain, by subsequent irradiation up to $550 \mathrm{~s}$, sequential uniform elongation from 37 to $45.5 \mathrm{~nm}$ and uniform shrinkage from 14 to $9.3 \mathrm{~nm}$ are proceeded. It has also been observed that MWCNT evolution rate is accelerated as it is thinned down to $11.5 \mathrm{~nm}$, shown in Fig. 1i-I. Nevertheless, overall, during irradiation, e-beam current density and curvature effect are almost remained uniform over the whole irradiated length of the MWCNT thus activating uniform elongation and shrinkage. Although under the increased irradiation, defect fabrication rate in the tube shells is increased steadily, MWCNT structural integrity is remained intact. This can be endorsed to the fact that dynamic competition between fabrication and annihilation processes of atomic defects is surpassed by the atomic annihilation process, while $\mathrm{sp}^{2}$ hybridized bonded network of multi-shells of MWCNT 
Fig. 1 Consecutive bright field TEM images (a-l) showing uniform elongation and shrinkage processes in 14-nm-thick and 37-nm-long MWCNT under increased irradiation time (shown at left bottom corner of each frame), when irradiated by uniform current density $\left(\sim 160 \mathrm{~A} \mathrm{~cm}^{-2}\right)$ of e-beam and beam spot diameter $200 \mathrm{~nm}$ expanding over a zone greater than the observed area

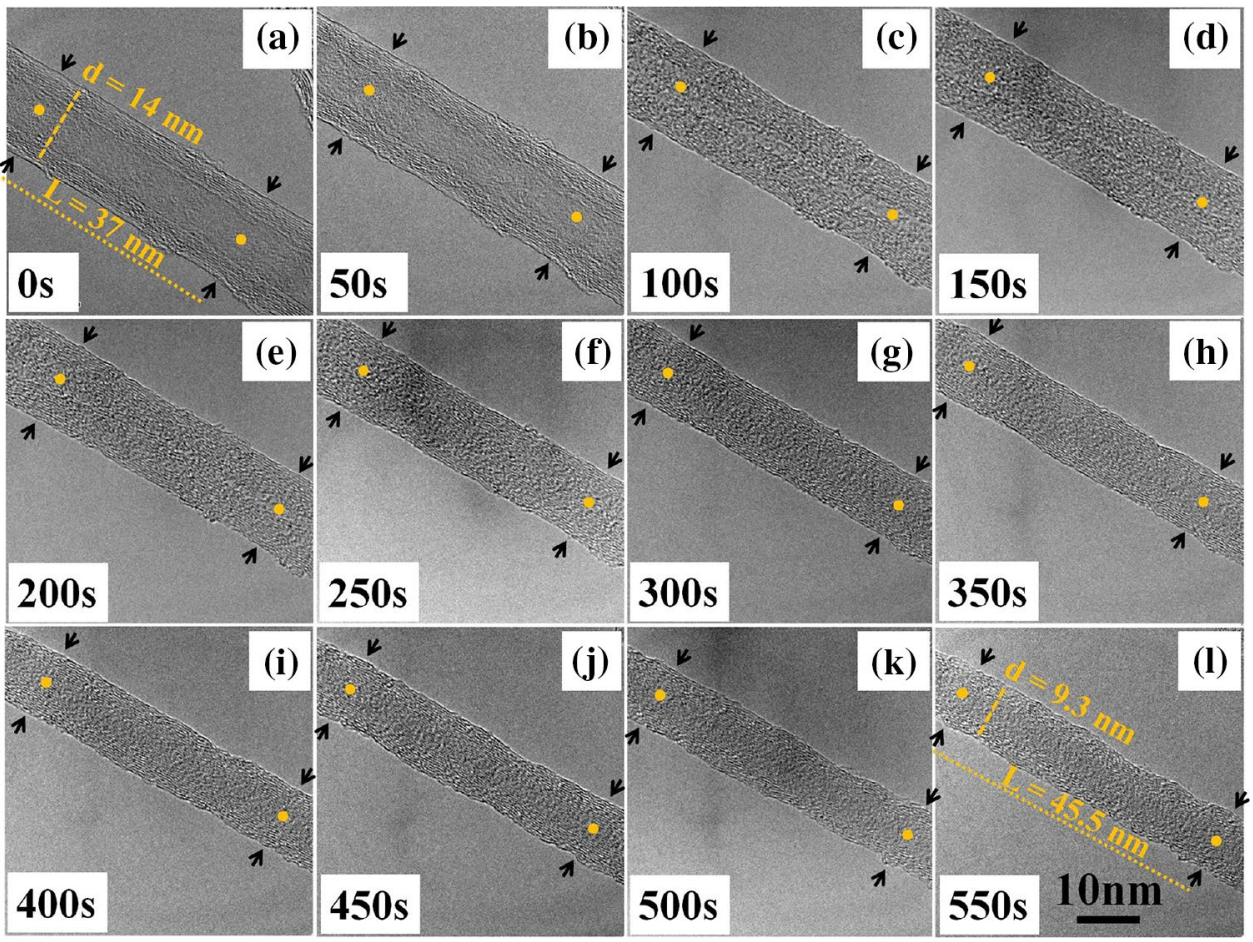

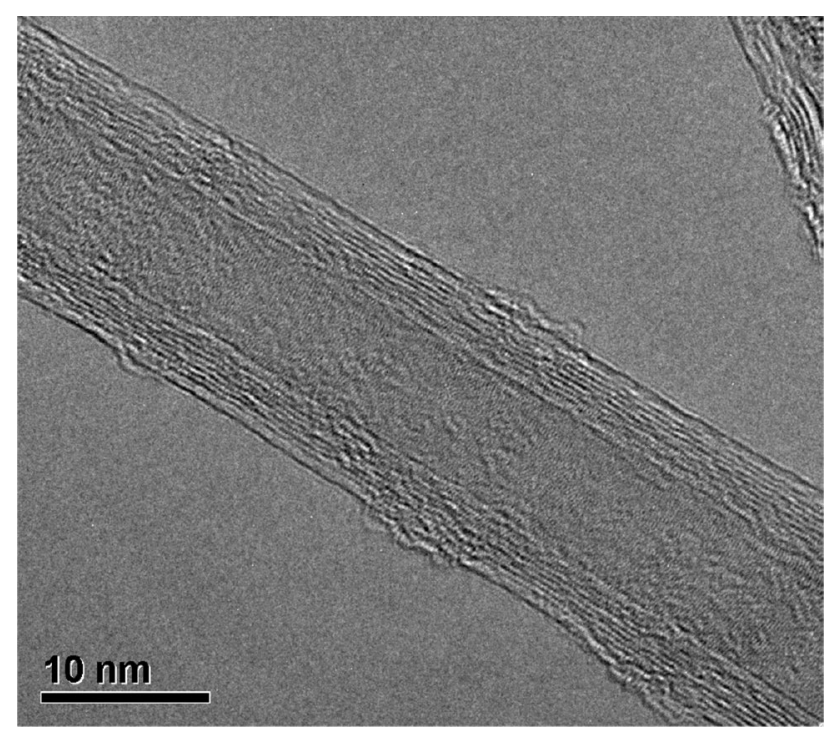

Fig. 2 A magnified TEM micrograph of the pristine MWCNT (before irradiation) shown in Fig. 1a. This illustrates the straight multi-shells and perfect geometry of the MWCNT

tends to restore its broken bonds via the self-healing ability. Such a MWCNT transformation has not been reported before. This remarkable transformation is as distinctive as uniform elongation and shrinkage induced under the uniform irradiation, where role of uniform nanoscaled curvature (surface energy) effect of the MWCNT is crucial. Thus, this can be called as uniform structural transformation. The consistency in the results has been confirmed by irradiating several identical MWCNT segments under the same irradiation conditions. It is assumed that thin enough MWCNTs under uniform beam current density produce the best results.

In addition, a slight amorphous carbon (contamination) over the outer surface of tube is visible in Fig. 1a that is unstable as it rapidly polishes off (or mixes) during irradiation up to $100 \mathrm{~s}$, seen in Fig. 1c and thus does not affect the structural evolution process at all. This amorphous carbon attachment is typical to the higher surface energy of MWCNT. Indeed, the irradiated MWCNT is the cleanest one as the magnified TEM micrograph in Fig. 2 illustrates that MWCNT possesses to about ten straight shells, perfect crystalline features, and well-defined cylindrical structure before irradiation. A small defects coarsening by accretion of carbon atoms between the contiguous wall gaps or by immobility of vacancies for extended period at low temperature or by both under prolonged irradiation [16] is inevitable. This can be attributed either to the minor fluctuation in the beam current density or non-uniformity in local atomic structure. Nevertheless, a substantial accumulation of local structural defects has not been viewed. This states that uniform diffusion and flow (transportation) of carbon atoms along the tube shells in the tube axis direction (elongation) and self-contraction (inward diffusion) of shells (shrinkage) are the ultrafast processes driven by the nanoscaled negative (positive) curvature effect under ultrafast e-beam activation at RT. 
Fig. 3 a The uniform elongation of the MWCNT with increasing irradiation time estimated by Fig. 1. To trace the successive elongations, MWCNT length is approximated as the length of the irradiated tube segment between the two yellow feature dots marked in Fig. 1; b The uniform shrinkage of the MWCNT diameter (radius) with increasing irradiation time approximated by Fig. 1. The fitting result demonstrated by the dashed line is obtained by implementing the equation shown below the data points, where value of the constant $b$ is $\sim 4.2$; c The MWCNT diameters traced every $6 \mathrm{~nm}$ along the tube length in Fig. 11 (between the two yellow dots, left dot at $0 \mathrm{~nm}$ and right dot at $45.5 \mathrm{~nm}$ )

Figure 3 illustrates the analogous plots for MWCNT elongation and shrinkage against irradiation time in addition to the point-to-point evaluation along the MWCNT length. By Fig. 3a, MWCNT elongation from 37 to $45.5 \mathrm{~nm}$ is averaged to $\sim 4 \times 10^{-3} \mathrm{~nm} / \mathrm{s}$, while by Fig. 3b, MWCNT shrinkage from 14 to $9.3 \mathrm{~nm}$ is averaged to $1.2 \times 10^{-2} \mathrm{~nm} / \mathrm{s}$. Obviously, during elongation, reduction in the MWCNT diameter is a quite natural mechanism and this phenomenon can be correlated with the Poisson's ratio that is calculated to be $\sim 1.5$. At that point, the MWCNT expands quite uniformly in the axial direction in the area marked between the two yellow dots in Fig. 11 . For this particular length $(\sim 45.5 \mathrm{~nm})$, on average, the reduction in the diameter is about $33 \%$ and approximately uniform shown in Fig. $3 \mathrm{c}$ that demonstrates point-to-point assessment along the MWCNT length. By quantitative analysis, assuming constant MWCNT volume, the significant uniform elongation is approximated to $23 \%$ for this (33\%) limited shrinkage. The elongation and the corresponding shrinkage are accelerated from 350 to $550 \mathrm{~s}$ of irradiation thus showing increasingly faster trends or nonlinear tendencies.

By thermodynamics, during the uniform elongation/ shrinkage, uniform diffusion of internal carbon atoms along the tube shells in the tube axis direction is driven by the nanoscaled curvature (high surface energy) of MWCNT. By taking into account the thermodynamic driving forces, shrinkage in the fixed length $L_{1}$ is precisely tracked by a new model [16] established about how MWCNT surface energy influences the shrinkage and correlates the kinetic relationship between the MWCNT shrinkage and the irradiation time,

$h\left(\sum_{i=1}^{n} r_{i}^{-b}+e\right)=-n \frac{\mathrm{d} r}{\mathrm{~d} t}$

Here, in Eq. 1, $h=2 \mathrm{Ka} / \mathrm{J}, e=F_{\mathrm{o}} / 4 \pi a L$, and $K=K_{\mathrm{o}} \exp \left(-\Delta G^{*} / R T\right)$, where $K_{\mathrm{o}}, R, T$, and $\Delta G^{*}$ are, respectively, force constant, gas constant, temperature, and activation energy for the MWCNT shrinkage. The surface energy factor $a$ is related to the activation energy
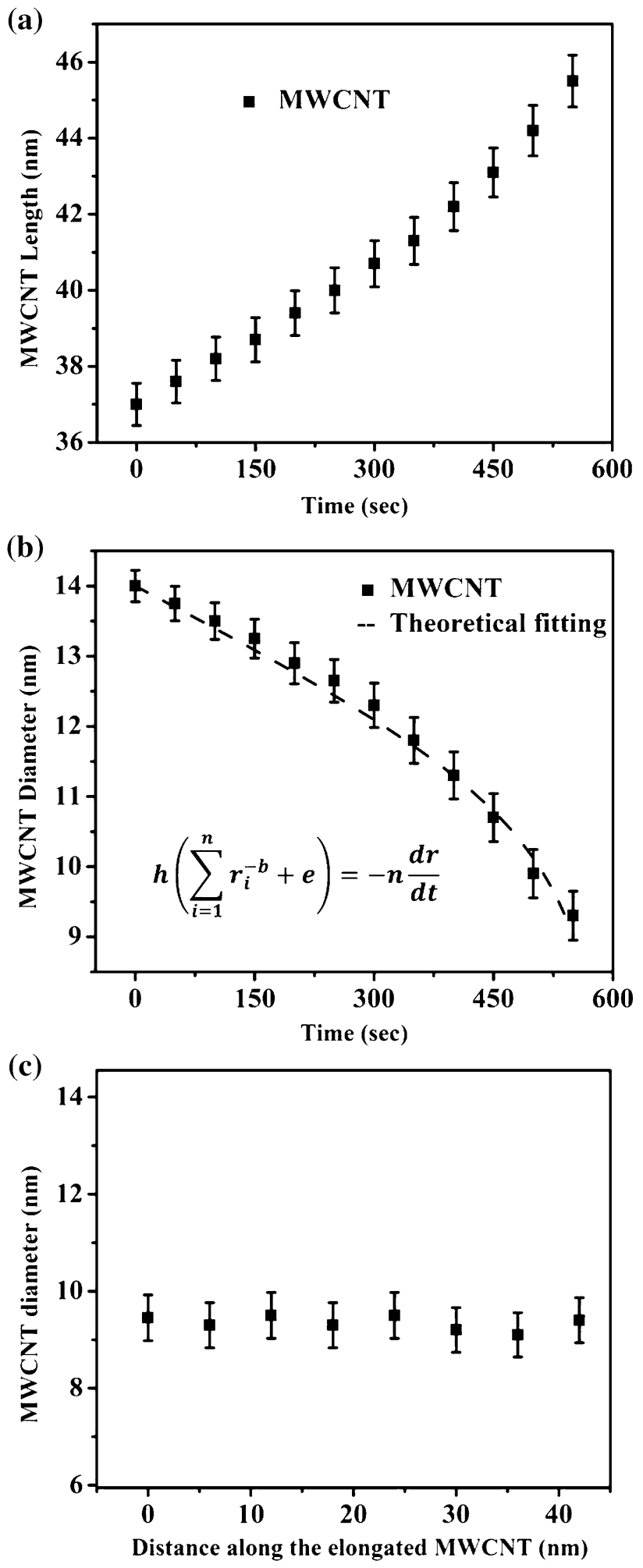

of the shrinkage, $J$ is the real density of carbon atoms in each shell, and $F_{\mathrm{o}}$ denotes Vander Waals' forces between neighboring shells, $n$ is total number of shells in the MWCNT, $r_{i}$ is radius of the ith shell, and $b$ is a constant representing curvature effect on the surface energy. A 
well-fitting result of the shrinkage process of MWCNT diameter (radius) under optimum beam current density of $160 \mathrm{~A} \mathrm{~cm}^{-2}$ and physical meaningful constants $b$ and $\Delta \mathrm{G}^{*}$, ranging from 14 to $9.3 \mathrm{~nm}$ under $550 \mathrm{~s}$, is obtained, shown by a dashed line in Fig. $3 \mathrm{~b}$. The result shows a theoretically fitting nonlinear curve of the experimental data for the value of constant $b$ at $4.2 \pm 0.4$. The uncertainty $( \pm 0.4)$ in the fitting curve arisen during the measurements is accounted in the error bars shown in Fig. 3b. This implies the effect of an additional nanoscaled curvature on surface energy of MWCNT to be proportional to $1 / r^{4.2}$ instead of $1 / r^{2}$, described in the previous models [19-23]. This variation clearly demonstrates a considerable increase in nanoscaled surface energy induced by the increased curvature of the shrunk MWCNT under e-beam irradiation.

In fact, the surface energy (curvature) effect in SWCNTs and MWCNTs has not been described in detail in the previous models [19-23]. For a static SWCNT, without considering the e-beam activation effect, calculations have been approximated on equilibrium, symmetrical, periodical, and linear nature of carbon nanostructures that cannot account the well-defined nanoscaled surface energy effect. Although, in the modified form of Cauchy-Born rule, inter-atomic potential in continuum analysis has been encompassed and additional surface energy of SWCNT has been predicted to be proportional to $1 / r^{2}$ [16]; effect of many body potentials among the carbon atoms has not been replicated clearly. Some local density approximations (simulations) [21-23] have also been failed to determine the net large scale electronic disorder of SWCNT. Furthermore, similar results $\left(1 / r^{2}\right)$ have also been obtained through generalized gradient approximation (GGA) by the ab initio method [19]. However, focus of the GGA has been remained on primary gradient of electronic density but not on the net large scale electronic disorder of SWCNT. As these factors have not been accounted in those simulations or the theoretical predictions, and thus, value of $b$ in our experimental results is larger than them.

In contrast to the Poisson's ratio (1.5) calculated herein, the computed values of the Poisson's ratio for MWCNTs and SWCNTs have, respectively, been approximated from 0.15 to 0.28 and 0.19 to 0.34 , demonstrated in the previous studies $[24,25]$. This indicates much higher effect of an additional nanoscaled curvature on the uniform radial shrinkage of MWCNT, which eventually leads toward uniform axial elongation. It has been investigated by computational study of the elasticity of a MWCNT that MWCNT elongation under tensile loading depends upon number of shells, and its Young's modulus varies from 4.70 (for a single shell) to $1.13 \mathrm{TPa}$ (for 10 shells) [25]. Furthermore, it has also been modeled that radial modulus of MWCNT increases with the increase in number of shells, decreases with the increase in MWCNT diameter and does not depend upon the Poisson's ratio [26]. That theoretical evaluation about the dependence of radial Young's modulus upon number of MWCNT shells agrees qualitatively well with our experimental results of the radial shrinkage, plotted in Fig. 3b. To compare, the radial shrinkage process in MWCNT delays, when a number of shells of the MWCNT are greater in number. And the radial Young's modulus sharply increases with the decrease in diameter of MWCNT for constant number of shells. This is consistent with our results for the MWCNT as well as for the SWCNT $[16,26]$. This is acceptable if we take into account the contributions of the bond length shortening and bond angle changes to the surface energy of MWCNT under the nanoscaled curvature effect $[16,25]$. Unfortunately, chiralities of the multiple-shells of MWCNT could not be predicted by our in situ TEM technique employed in the present study. The effect of chirality on the MWCNT deformation has been studied by ab initio method, and it has been anticipated that armchair CNT is energetically much more stable than that of other chiralities of the same radii. Nevertheless, this difference is extremely small and becomes narrow with the increase in CNT diameter [24].

Therefore, in view of the tremendous surface energy of the nanoscaled curvature effect, the structural evolution of the shrunk MWCNT under e-beam irradiation can be conceived as schematically drawn in Fig. 4. By e-beam irradiation, for irradiated length of each shell of MWCNT segment, the negative (positive) curvature effect becomes dynamic and drives (forces) each shell to shrink (diffuse inward) and to achieve smaller curvature of lower energy thus inducing diffusion and plastic flow (transportation) of enormous carbon atoms along the shells in the tube axis direction. The increased instability in the MWCNT under increased irradiation assists e-beam to enhance number density of carbon atoms to diffuse (flow) and escorts toward increased susceptibility (decreased mechanical strength) of MWCNT. This means that the continuous and uniform diffusion and flow (transportation) not only

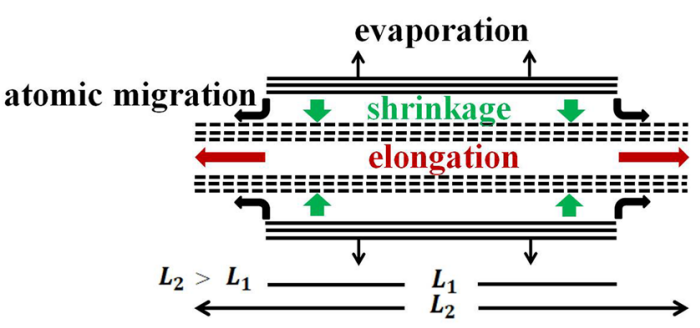

Fig. 4 Schematic demonstration of the uniform elongation process in MWCNT by atomic migration (elongation) along the tube shells in the tube axis direction and inward diffusion (shrinkage) of multishells in the radial direction 
prevents refilling of vacancies but also obstructs saturation of dangling bonds under the constant and uniform energy transfer by the high enough e-beam energy deposition rate $\sim 160 \mathrm{~A} \mathrm{~cm}^{-2}$. Meanwhile, the restoration aptitude of the curved $\mathrm{sp}^{2}$ hybridized structure of MWCNT pulls each shell to contract inward and increase the length and decrease the diameter while tube structure is attempting to achieve equilibrium. Few carbon atoms trapping between the contiguous shell gaps or their diffusion in the inner hollow or their direct evaporation into the free space through the vacancies are also possible. Essentially, the structural evolution process remains uniform as e-beam current density remains uniform. Since nanoscaled curvature of inner shells could be larger than that of outer shells thus resulting into their faster instability or faster shrinkage/breakage. This facilitates further shrinkage and speeds up self-contraction process of shells driven by their increased negative (positive) curvature effects, which enhance the evolution process, while on the other hand, diffusion and flow of carbon atoms continue along the shells in the tube axis direction.

Several earlier experimental and theoretical studies on the elongations of MWCNTs/SWCNTs [9-15], and amorphous (crystalline) nanowires [27-29] induced by the imposed strain with/without e-beam have been illustrated, but in a different perspective. It is important to mention here that our results in Fig. 1 are essentially dissimilar compared with those demonstrated in the previous reports [9-15, 27-29]. This is reasonable because elongation in our experiments indisputably rely on the intrinsic inward tensile stress of each shell of MWCNT (radial shrinkage) induced under the nanoscaled negative (positive) curvature effect and e-beam activation, which is different from the previous experiments, where an extrinsic force under e-beam irradiation has been employed to elongate the MWCNTs/SWCNTs and nanowires. This makes it too intricate to evaluate let alone the effect of e-beam irradiation. Such one dimensional nanostructures have been elongated by the imposed strain employed in the axial direction that is in fact not an elongation caused due to the radial shrinkage or by the e-beam irradiation. The elongations attained by this way are essentially different than our results as it is quite easy to elongate one dimensional nanostructure via an imposed strain than the elongation caused by radial shrinkage induced under uniform nanoscaled curvature effect and uniform e-beam irradiation. In fact, our results are dissimilar to the bulk materials and to the current literature [9-15, 27-29] as the current literature is based on classical knock-on mechanism and molecular dynamics simulations, the symmetrical, periodical, and linear nature of LDNs (approximations) in equilibrium, whereas the above MWCNT uniform elongation under e-beam at RT can be well underlined by the nanoscaled curvature effect and e-beam-induced soft modes and lattice instability effect as explained below.

As opposed to the bulk materials, the high surface to volume ratio of MWCNTs originates disorder in the atomic and electronic structure. This inherently shortens the bond lengths and transforms the carbon $(\mathrm{C}-\mathrm{C})$ bond angles thus confining the local electronic charges and dangling bonds quantum mechanically and leading toward localization and densification of bond energies through uplifting the pair potentials. Accordingly, a significant increase in the bond strains, bond strengths, and bond energies occurs which stores an additional energy in the $\mathrm{C}-\mathrm{C}$ bonds or around the structural defects thus eventually softening the phonons and reducing the 'Debye temperature (melting point). These salient features are sensitive to the nanoscale and become more pronounced in very thin MWCNTs [1, 16]. Furthermore, in contrast to the amorphous (crystalline) nanowires, metal nanoparticles, and bulk materials, MWCNT inner and outer surfaces disparately lead toward two distinct curvatures, a negative curvature at the inner surface and a positive curvature at the outer surface illustrated in Fig. 5a. The negative curvature effect becomes more prominent than that of the positive curvature effect at the nanoscale thus originating two diverse classes of phonons at the two separate surfaces $[1,16]$. By negative curvature, not only the inner surface energy of MWCNT increases dramatically but also MWCNT becomes susceptible to irradiation and each shell drives (shrinks) kinetically in the inward radial direction due presumably by the intrinsic non-equilibrium and nonlinear nature thus escorting toward the uniform shrinkage and uniform elongation.

This study is a follow-up of our previous report [1] which anticipates that a MWCNT of smaller diameter possesses enlarged negative curvature (higher surface energy) to induce atom instability, where MWCNT shrinkage (a)

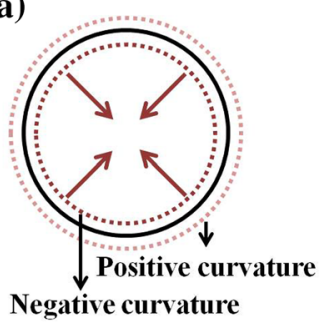

(b)

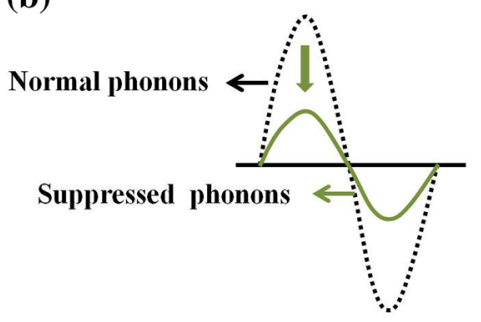

Fig. 5 Schematic demonstration of a the bent (away from the normal equilibrium positions) carbon atoms of SWCNT, where influence of the negative curvature (inner surface energy) to drive carbon atom instability becomes more pronounced than that of the positive curvature (outer surface energy) and $\mathbf{b}$ the phonon suppression in MWCNT illustrating that the corresponding suppression of phonons in the MWCNT is severer compared with the bulk counterparts, nanowire, and nanoparticles 
accelerates by the increased nanoscaled non-uniform negative curvature effect with thinning down of the tube and leads the tube structure toward non-uniform elongation and non-uniform shrinkage activated by the focused e-beam. However, in that case, a quantitative correlation between thermodynamic kinetic process of the accelerated shrinkage and the nanoscaled curvature effect has been too intricate to establish because of the probable refilling of the flow of atoms away/toward the necked region activated by another curvature of the nearby necked region along the wire axis direction that could intricate (halt) the thermodynamic kinetic process of the accelerated shrinkage of the necked region. Essentially, such a trend is always present when a MWCNT shrinks non-uniformly, and therefore, it cannot be estimated precisely while tracking the thermodynamic kinetics of the nearby necked zone under irradiation. Nevertheless, it can be assessed by the present results that a well-defined relationship between our proposed new surface energy model and uniform shrinkage of MWCNT is quantitatively established by precisely tracking the MWCNT uniform shrinkage kinetics induced under subsequent e-beam doses and by correlating with the MWCNT uniform elongation illustrated in Fig. 1. This has become possible only because the MWCNT geometry (shells) is remained straight or uniform in the axis direction during the entire irradiation period and MWCNT is elongated and shrunk smoothly and adequately. Therefore, effect of any non-uniform profile of the nanoscaled curvature of MWCNT can be avoided. Furthermore, a thin enough MWCNT is chosen to irradiate at the very initial stage and only a limited shrinkage is noted, while on the other hand, e-beam current density is being kept high enough and irradiation period is limited. Thus, during irradiation, fluctuations in the e-beam current density or in the structural evolution of tube can be discarded. Nevertheless, the quite natural reduction in diameter of MWCNT with elongation could be delayed by the reinforcing fact of the ten shells compared to the SWCNT. As the present study indicates that elongations of SWCNT, double-walled CNT, and triple-walled CNT are very possible; evaluating these elongations completely is an area for upcoming improvement. Eventually, neither experiments nor theoretical predictions have yet fully evaluated the effects of e-beam irradiation and nanoscaled curvature on instability of MWCNT. It can be speculated that when the nanoscaled negative curvature effect activates under e-beam, the tube shells shrink in the radial direction and drive carbon atoms; the carbon atoms flow along the tube shells in the tube axis direction and permanently elongate it.

Next, we evaluate the e-beam effects in activating the dramatic plastic elongation (diffusion and plastic flow of carbon atoms) observed. It noteworthy that any structural evolution in the MWCNT suspends under suspended irradiation even when the above uniform elongation process is once activated for time being. This obviously reveals the fundamental source of ultrafast soft modes and lattice instability effect in MWCNTs under e-beam illustrated in Fig. 1 and in other CNTs cases $[1,16]$. To attain insights into how e-beam activates soft modes and lattice instability at $\mathrm{RT}$, it can be considered that susceptibility (activeness) and dynamical response of carbon bonds to e-beam irradiation enhance drastically by the additional storage of energy in $\mathrm{C}-\mathrm{C}$ bonds as described above. This response is closely associated with the excitations of valence electrons that represent the bonding character $[1,16]$. Thus, this not only increases the energy of electronic states but also assists bond instability by softening the phonons under e-beam excitation. In fact, in condensed matter, phonon softening and bond instability by electron, laser, and ion beams are a universal phenomenon $[1,16]$ that is known as e-beaminduced soft mode and lattice instability effect, a totally different phenomenon than the thermal or knock-on phenomenon, where energy deposited by the ultrafast energetic electrons of the e-beam surpasses the normal modes of phonons (natural frequencies) of condensed matter (MWCNTs) $[1,16]$. Likewise, the energy deposition by ultrashort $\left(10^{-15} \mathrm{~s}\right)$ laser pulses, the energetic e-beam exchanges (deposits) energy with the $\mathrm{C}-\mathrm{C}$ bonds in an ultrafast or ultrashort time $(t \rightarrow 0)$ and in a non-equilibrium, extremely confined, precised, and localized way [ 1 , 16]. This originates a high density of soft modes thus distorting the $\mathrm{C}-\mathrm{C}$ (covalent) bonds of $\mathrm{sp}^{2}$ hybridized MWCNT structure and causing bond instabilities demonstrated in Fig. 5b. In this way, carbon atoms not only easily break the barrier imposed by the strong structural constraints and lose their parent positions but also become able to diffuse and flow (migrate) uniformly and plastically along the shells in the tube axis direction.

\section{Conclusion}

In conclusion, it can be contemplated that the typically brittle thin enough MWCNTs, when used in nanotechnology (or assembled into nanodevices), can be uniformly elongated (post-processed) by high enough intensity e-beam irradiation at RT. This is because the energetic e-beam will reduce the energy barrier throughout the whole irradiated MWCNT length to facilitate the curvature effect, which impart driving force to the tube shells, for their uniform inward diffusion and uniform plastic elongation in the axial direction. This inward diffusion induces the carbon atomic flow that activates shrinkage, while carbon atoms migrate in the tube axis direction along the shells. Uniform plastic elongation is thus 
achieved at RT, approximately equivalent to the macroscaled tubes. In addition, when initially the MWCNT is thin enough and shrinkage (or elongation) is limited, it will be adequately feasible to establish the kinetic relationship between the shrinkage and irradiation time that is required to quantitatively track the shrinkage process to account the nanoscaled additional curvature effect of the MWCNT. This fitting result illustrates emergence of surface energy induced by nanoscaled curvature effect that is much greater compared with the theoretical predictions and simulations. Otherwise stated, a significant uniform plastic elongation mechanism dependable upon the imposed strain [9-15] is now made pronounced at RT by atomic diffusion and flow activated by e-beam induced soft modes and lattice instability effect and nanoscaled curvature effect.

Acknowledgements We highly acknowledge the support and suggestions for TEM observation by Qiming Hong from electron microscopy center, Xiamen University.

Funding This work was supported by the National Key Research and Development Program of China (2016YFB0400801, 2016YFB0400800), 863 program (2014AA032608), National Natural Science Foundation of China (U1405253, 61227009, 90921002), Natural Science Foundation of Fujian Province of China (2016J01265), Guangdong Natural Science Foundation (2018A0303130271), and Fundamental Research Funds for the Central Universities (20720160015, 20720180014).

\section{Compliance with ethical standards}

Conflict of interest The authors declare that they have no conflict of interest.

\section{References}

1. Khan I, Bing H, Huang SL, Wu CX (2018) Multi-walled carbon nanotubes under focused electron beam: metal passivation effect and nanoscaled curvature effect. J Phys Condens Matter 30:385302

2. Liu B, Ren W, Liu C, Sun CH, Gao L, Li S, Jiang C, Cheng HM (2009) Growth velocity and direct length-sorted growth of short single-walled carbon nanotubes by a metal-catalyst-free chemical vapor deposition process. ACS Nano 3:3421-3430

3. Treacy MMJ, Ebbesen TW, Gibson JM (1996) Exceptionally high Young's modulus observed for individual carbon nanotubes. Nature 381:678-680

4. Wong EW, Sheehan PE, Lieber CM (1997) Nanobeam mechanics: elasticity, strength, and toughness of nanorods and nanotubes. Science 277:1971-1975

5. Yakobson BI, Brabec CJ, Bernholc J (1996) Nanomechanics of carbon tubes: instabilities beyond linear response. Phys Rev Lett 76:2511-2514

6. lijima S (1991) Helical microtubules of graphitic carbon. Nature 354:56-58

7. Iijima S, Ichihashi T (1993) Single-shell carbon nanotubes of $1 \mathrm{~nm}$ diameter. Nature 363:603-605
8. Hamada N, Sawada S, Oshiyama A (1992) New one dimensional conductors: graphitic microtubules. Phys Rev Lett 68:1579-1581

9. Sato M (2011) Elastic and plastic deformation of carbon nanotubes. Proc Eng 14:2366-2372

10. Yu M-F, Lourie O, Dyer MJ, Moloni K, Kelly TF, Ruoff RS (2000) Strength and breaking mechanism of multiwalled carbon nanotubes under tensile load. Science 287:637-640

11. Arias I, Arroyo M (2008) Size-dependent nonlinear elastic scaling of multiwalled carbon nanotubes. Phys Rev Lett 100:085503

12. Kim H-I, Wang M, Lee SK, Kang J, Nam J-D, Ci L, Suhr JW (2017) Tensile properties of millimeter long multi-walled carbon nanotubes. Sci Rep 7:9512

13. Li F, Cheng HM, Bai S, Su G (2000) Tensile strength of singlewalled carbon nanotubes directly measured from their macroscopic ropes. Appl Phys Lett 77:3161-3163

14. Yakobson BI (1998) Mechanical relaxation and "intramolecular plasticity" in carbon nanotubes. Appl Phys Lett 72:918-920

15. Huang JY, Chen S, Wang ZQ, Kempa K, Wang YM, Jo SH, Chen G, Dresselhauss MS, Ren ZF (2006) Superplastic carbon nanotubes. Nature 439:281

16. Khan I, Huang SL, Wu CX (2017) Multi-walled carbon nanotube structural instability with/without metal nanoparticles under electron beam irradiation. New J Phys 19:123016

17. Wittke JH (2006) Class notes: introduction to micro-beam techniques (Northern Arizona University). https://www2.nau.edu/ microanalysis/wordpress/index.php/introduction/\#Introducto rycomments

18. Banhart F (1999) Irradiation effects in carbon nanostructures. Rep Prog Phys 62:1181-1221

19. Gulseren O, Yildirim T, Ciraci S (2002) Systematic ab initio study of curvature effects in carbon nanotubes. Phys Rev B 65:153405-1-153405-4

20. Jiang H, Zhang P, Liu B, Huang Y, Geubelle PH, Gao H et al (2003) The effect of nanotube radius on the constitutive model for carbon nanotubes. Comput Mater Sci 28:429-442

21. Robertson DH, Brenner DW, Mintmire JW (1992) Energetics of nanoscale graphitic tubules. Phys Rev B 45:12592-12595

22. Sanchez-Portal D, Artacho E, Soler JM (1999) Ab initio structural, elastic, and vibrational properties of carbon nanotubes. Phys Rev B 59:12678-12688

23. Ye LH, Liu BG, Wang DS (2001) AB initio molecular dynamics study on small carbon nanotubes. Chin Phys Lett 18:1496-1499

24. Yakobson Bl, Avouris P (2001) Mechanical properties of carbon nanotubes. In: Dresselhaus G, Avouris P (eds) Carbon nanotubes, Topics applied physics, vol 80. Springer, Berlin, Heidelberg, pp 287-327

25. Tu Z-C, Ou-Yang Z-C (2002) Single-walled and multiwalled carbon nanotubes viewed as elastic tubes with the effective Young's moduli dependent on layer number. Phys Rev B 65:233407

26. Dai XB, Merlitz H, Wu CX (2006) Transverse elasticity of multiwalled carbon nanotubes. Eur Phys J B 54:109-112

27. Zheng H, Liu Y, Mao SX et al (2012) Beam-assisted large elongation of in situ formed $\mathrm{Li}_{2} \mathrm{O}$ nanowires. Sci Rep 2:1-4

28. Han X, Zheng K, Zhang $Y$ et al (2007) Low-temperature in situ large-strain plasticity of silicon nanowires. Adv Mater 19:2112-2118

29. Zheng K, Wang C, Cheng YQ et al (2010) Electron-beam assisted superplastic shaping of nanoscale amorphous silica. Nat Commun 1:24

Publisher's Note Springer Nature remains neutral with regard to jurisdictional claims in published maps and institutional affiliations. 